\title{
CARACTERÍSTICAS ANTROPOMÉTRICAS Y POTENCIA DE MIEMBROS INFERIORES EN JUGADORES UNIVERSITARIOS DE RUGBY-7
}

\author{
de Azevedo Martins, Moisés Silvestre; Nunes, Eryclis Miguel; Rodrigues, Carlos Diego; Hernández- \\ Mosqueira, Claudio; Fernandes da Silva, Sandro \\ CARACTERÍSTICAS ANTROPOMÉTRICAS Y POTENCIA DE MIEMBROS INFERIORES EN JUGADORES \\ UNIVERSITARIOS DE RUGBY-7 \\ MHSalud, vol. 15, núm. 2, 2018 \\ Universidad Nacional, Costa Rica \\ Disponible en: http://www.redalyc.org/articulo.oa?id=237056017006 \\ DOI: https://doi.org/10.15359/mhs.15-2.4
}

Esta obra está bajo una Licencia Creative Commons Atribución-NoComercial-SinDerivar 3.0 Internacional. 


\section{CARACTERÍSTICAS ANTROPOMÉTRICAS Y POTENCIA DE MIEMBROS INFERIORES EN JUGADORES UNIVERSITARIOS DE RUGBY-7}

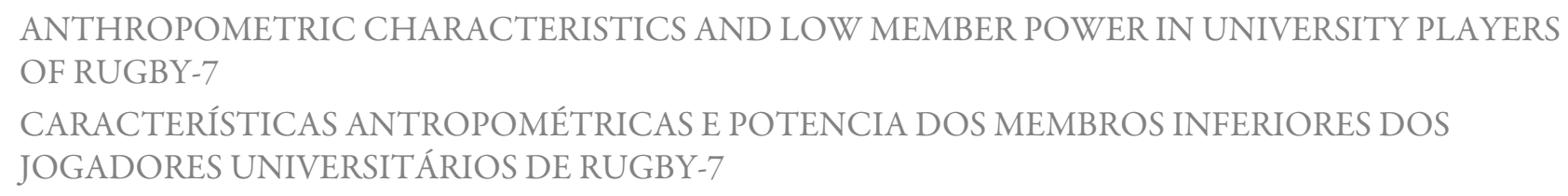

Moisés Silvestre de Azevedo Martins

Universidade Federal de Lavras, Brasil

moisesdreamcast72@gmail.com

(D) http://orcid.org/0000-0001-7116-0579

Eryclis Miguel Nunes

Universidade Federal de Lavras, Brasil

erycliseduardo25@hotmail.com

(iD http://orcid.org/0000-0003-0263-3429

Carlos Diego Rodrigues

Universidade Federal de Lavras, Brasil

dgndiego@hotmail.com

\section{Claudio Hernández-Mosqueira}

Universidad de los lagos, Chile

claudio.hernandez@ulagos.cl

(D) http://orcid.org/0000-0001-9392-2319

Sandro Fernandes da Silva

Universidade Federal de Lavras, Brasil

sandrofs@def.ufla.br

(D) http://orcid.org/0000-0003-0516-6408
DOI: https://doi.org/10.15359/mhs.15-2.4

Redalyc: http://www.redalyc.org/articulo.oa? $\mathrm{id}=237056017006$
Recepción: 05 Marzo 2018

Aprobación: 01 Agosto 2018

\section{Resumen:}

El objetivo de este estudio fue comparar las variables antropométricas y de potencia de miembros inferiores (PMI) en una muestra de jugadores de rugby- 7 de diferentes posiciones. Participaron 22 jugadores universitarios clasificados de acuerdo con las posiciones ocupadas en el terreno de juego en back y forward. Para evaluar el porcentaje de grasa, se utilizó el protocolo de 7 pliegues cutáneos de Jackson \& Pollock, y para las pruebas de PMI salto vertical (CMJ), salto horizontal y salto triple. Con el afán de comparar las variables del estudio, fue adoptado el test $\mathrm{T}$ para muestras pareadas, siendo el $\mathrm{p}<0,05$. Los resultados indicaron una diferencia significativa entre la masa total $(\mathrm{t}=, \mathrm{p}<0,01)$, la masa libre de grasa $(\mathrm{p}<0,05)$, la masa adiposa $(\mathrm{p}<0,01)$ y el CMJ $(\mathrm{p}<0,005)$ entre los grupos. Se observa que las características antropométricas y neuromusculares para los forwards son distintas de las de los backs, debido a la posición de juego, que requiere una configuración morfológica y física distinta.

Palabras CLAVE: rugby, potencia de miembros inferiores, variables antropométricas, universidad.

\section{Abstract:}

The objective of this study was to compare the anthropometric and lower limb power (PMI) variables in a sample of rugby-7 players holding different positions. Twenty-two rugby-7 college players participated; according to the positions occupied on the playing field, they were classified in back and forward. The protocol of seven skin folds of Jackson \& Pollock was used to evaluate the percentage of fat; and for the PMI tests vertical jump (CMJ), horizontal jump and triple jump. The $\mathrm{T}$-test for paired samples 
was adopted, being $\mathrm{p}<0.05$, to compare the study variables. The results indicated a significant difference between the total mass $(\mathrm{p}<0,01)$, the fat-free mass $(\mathrm{p}<0,05)$, the fat mass $(\mathrm{p}<0,01)$, and the CMJ $(\mathrm{p}<0,005)$ between the groups. It is observed that the anthropometric demands for the forwards are different from the backs, due to the characteristic of the game position, which requires a different physical and morphological configuration.

KEYWORDS: rugby, power of lower limbs, anthropometric variables, college students.

\section{Resumo:}

O objetivo deste estudo foi comparar as variáveis antropométricas e de potência dos membros inferiores (PMI) em uma amostra de jogadores de rúgbi-7 de diferentes posições. Participaram 22 jogadores universitários, classificados de acordo com as posições ocupadas no campo de jogo para adiante e para trás. Para avaliar o percentual de gordura, utilizou-se o protocolo de 7 dobras cutâneas de Jackson \& Pollock, e para os testes de PMI salto vertical (CMJ), salto horizontal e salto triplo. Com o objetivo de comparar as variáveis do estudo, o teste $T$ foi adotado para amostras de dupla, sendo $\mathrm{p}<0,05$. Os resultados indicaram uma diferença significativa entre a massa total $(t=, p<0,01)$, a massa livre de gordura $(p<0,05)$, a massa adiposa $(p<0,01)$ e o CMJ $(\mathrm{p}<0,005)$ entre os grupos. Observa- se que as características antropométricas e neuromusculares para os atacantes são diferentes daquelas das costas, devido à posição de jogo, que requer uma configuração morfológica e física diferente.

PALAVRAS-CHAVE: rugby, poder dos membros inferiores, variáveis antropométricas, universidade.

\section{INTRODUCCIÓN}

El rugby-7 es una modalidad practicada en todo el mundo, siendo una rama del rugby tradicional. Se caracteriza por ser colectivo, de invasión, y tiene el predominio de alta intensidad de esfuerzo (Sousa et al., 2016). Es practicado con 7 jugadores en lugar de 15, con una duración de 2 tiempos de 7 minutos (Navarro-Zurita, Gálvez, López y Suárez-Arrones, 2017). Los campeonatos se distribuyen en 2 días y las dimensiones del campo de juego son las mismas del juego tradicional $(100 \times 70)$. El deporte ya está incluido en multi-competiciones deportivas, incluyendo los Juegos Asiáticos, Juegos del Pacífico, Juegos de la Commonwealth, Juegos Mundiales Universitarios, y, en el 2016, marcó el regreso del rugby a las olimpiadas (World Rugby, 2014).

Los equipos de rugby-7 son formados por 4 backs (defensores) y 3 forwards (atacantes). Los backs son los encargados de romper la defesa adversaria y marcar puntos, se valen de la velocidad en la carrera y pases largos, teniendo como atributos la rapidez, agilidad, bajo porcentaje de grasa y una capacidad aeróbica (VO2MÁX) muy desarrollada (Dacres-Mannings, Rochester y Frail, 2001). Los forwards se encargan de realizar los scrums, lineouts y garantizar la posesión del balón, necesitan ser más fuertes y pesados para soportar el impacto de las formaciones, por lo que presentan una estatura, masa muscular y porcentaje de grasa mayor que los backs (Scott, Roe, Coats y Peipoli, 2003).

Sobre las capacidades físicas, el rugby-7 presenta una gran demanda, en la que se destacan velocidad; fuerza máxima; potencia; agilidad; flexibilidad; tiempo de reacción; resistencia aeróbica y anaerobia; resistencia de fuerza y coordinación motora (Duthie, Pyne y Hooper, 2003; Haff y Nimphius, 2012; Monteiro, Mendes, Paz, Miranda y Lima, 2015; Roe, Darral-Jones, Till y Jones, 2016). Entre estas capacidades, se destaca la potencia de miembros inferiores (PMI), porque en este juego la manifestación de fuerza ocurre en ellos. Dada la importancia de la PMI en el desempeño del rugby, estudios previos resaltan la importancia de valorar la potencia muscular en sus jugadores y, aún más, de conocer las diferencias entre las posiciones, pero todavía es escasa de evaluaciones la PMI en diferentes posiciones de juego (Caneviski, Crepaldi y Fernandes, 2017; Cronin y Sleivert, 2005; Dinardi, Andrade e Ibiapina, 2017; De Lacey, Brughelli, McGuigan y Hansen, 2014; Leite et al., 2016; Loturco et al., 2017).

De acuerdo con lo mencionado y con el fin de incrementar la información disponible, el objetivo del presente estudio es comparar las variables antropométricas y de PMI entre diferentes posiciones de juego en un grupo de jugadores de rugby- 7 . 


\section{Materiales y MÉTODOS}

\section{Diseño}

Se trata de un estudio descriptivo y comparativo. Participaron 22 jugadores de equipos de rugby- 7 masculinos universitarios de la ciudad de Lavras, Brasil, divididos en 2 grupos: los backs ( $\mathrm{n}=11$, masa $=80,22 \pm 2,97$ $\mathrm{kg}$, estatura $=180 \pm 0,01 \mathrm{~cm}$, masa adiposa $=13,27 \pm 1,41 \%$, masa libre de grasa $=68,54 \pm 2,62 \mathrm{~kg}) \mathrm{y}$ los forwards $(\mathrm{n}=11$, masa $=97,37 \pm 2,72 \mathrm{~kg}$, estatura $=181 \pm 0,01 \mathrm{~cm}$, masa adiposa $=19,21 \pm 1,41 \%$, masa libre de grasa $=78,32 \pm 1,37 \mathrm{~kg}$ ). Todos los jugadores participaron de forma voluntaria y debían ser universitarios practicantes de rugby-7, tener por lo menos 1 año de experiencia en la modalidad, no presentar patologías que interfieran en el movimiento del salto y no jugar o haber jugado en la selección brasileña de rugby-7. La presente investigación fue aprobado por el Comité de Ética en Investigación con Seres Humanos de la Universidad Federal de Lavras (CAAE 54883616.2.0000.5148).

\section{Procedimientos}

El trabajo se dividió en 2 días; el primero, los participantes inicialmente se orientaron sobre lo que ocurría en los procedimientos del estudio, debieron firmar un término de consentimiento libre y esclarecido (TCLE), de acuerdo con la resolución 512/16 del Consejo Nacional de Salud de Brasil. En seguida, se realizó una evaluación antropométrica mediante la cual se midió la estatura, la masa y los valores de 7 pliegues cutáneos, acordes con el protocolo de Jackson y Pollock (1978), para la evaluación del porcentaje de masa adiposa y masa libre de grasa.

Al día siguiente, los evaluados se sometieron a un calentamiento general y específico para miembros inferiores, que tenía 5 minutos de duración. A continuación, se realizó el test de salto horizontal con 3 intentos, con un tiempo de descanso de 30 segundos entre los intentos y 1 minuto al final de la prueba. Luego se llevó a cabo el test de salto triple, con 1 minuto de descanso al final y se cerró con el test de salto vertical (CMJ), ejecutando 3 intentos con 1 minuto de recuperación pasiva entre cada uno. La elección de estos test comprende la batería de pruebas de potencia, propuesta por la Confederação Brasileira de Rugby (2014), creada con el fin de estandarizar las evaluaciones entre los clubes brasileños.

\section{Antropometría}

Se midió la estatura y masa corporal, utilizando una balanza con precisión $0,1 \mathrm{~kg}$ (Welmy ${ }^{\circ}$ ) y un estadiómetro con resolución de $0,1 \mathrm{~cm}$ (Welmy ${ }^{\circ}$ ). Para la estimación del porcentaje de grasa, se midieron los pliegues subescapular, tricipital, pectoral, axilar-media, suprailíaca, abdominal y muslo, utilizando un plicómetro científico (marca Cescorf $)$; para determinar el porcentaje de grasa, se utilizó la siguiente ecuación: DC Hombres Adultos $=1,11200000-\left[0,00043499(S T)+0,00000055(S T)^{2}\right]-[0,0002882$ (edad) $]$, donde ST es la suma de los pliegues cutáneos. Para el coeficiente de masa magra, se utilizó la fórmula: masa - masa grasa (Jackson y Pollock, 1978).

\section{Salto triple}

Se utilizó el protocolo de pruebas de la Confederação Brasileira de Rugby (2014), que consiste en realizar 3 saltos consecutivos, usando los brazos para dar impulso, tratando de alcanzar la mayor distancia posible. El primer salto se hace con los pies en paralelo detrás de la línea de salida y no se puede saltar con 1 pie en los 
otros 2 saltos. La distancia de este salto fue medida con una cinta métrica extendida en el suelo, desde la línea de salida hasta el punto del talón, y se empleó como criterio la media de los 3 intentos.

\section{Salto horizontal}

Se utilizó el protocolo de Haunsicker y Reiff (1976), con el cual cada individuo debe realizar 3 intentos con un descanso de 30 segundos entre uno y otro. La prueba consiste en saltar la mayor distancia posible en 1 solo salto, usando los brazos para dar impulso; el salto debe realizarse con los pies paralelos detrás de la línea de salida. La distancia del salto se midió con una cinta métrica extendida en el suelo, a partir de la línea de salida hasta el talón, y se empleó como criterio la media de los 3 saltos.

\section{Salto vertical (CMJ)}

Se usó lo propuesto en el protocolo de Bosco y Riu (1994). El evaluado se coloca de pie sobre la plataforma de contacto, con las manos en la cintura, los pies al ancho de los hombros y en completo contacto con la plataforma, el tronco erguido y las rodillas en extensión completa. Al estímulo sonoro pronunciado por el evaluador, el individuo realiza un salto, que busca alcanzar su altura máxima. En total se ejecutaron 3 intentos y fue considerada para el estudio la mejor marca; entre cada uno de los intentos, el evaluado tuvo 1 minuto para recuperación pasiva. El salto fue realizado en una plataforma de contacto de la marca CEFISE* El nivel de confiabilidad de jugadores de rugby en el CMJ es de R2 = 0,987 (Dobbin, Hunwicks, Highton y Twist, 2017).

\section{Análisis de los datos}

Para analizar los datos, se utilizó estadística descriptiva (media y desviación estándar), e inferencial. Se comprobó la normalidad de aquellos a través de la prueba Shapiro-Wilk. Con el propósito de comparar los grupos, se realizó la prueba $\mathrm{T}$ de Student para muestras pareadas. En el procesamiento estadístico de la información, se usó el programa informatizado SPSS en su versión 21.0. Para todos los análisis, se adoptó un nivel de significancia de $\mathrm{p}<0,05$.

\section{Resultados}

En la tabla 1, se presentan las diferencias estadísticamente significativas $\left(^{*}\right)$ en las variables de masa total ( $\mathrm{p}$ $=.0012)$, masa adiposa $(\mathrm{p}=.019)$ y masa libre de grasa $(\mathrm{p}=.005)$ entre los grupos backs y forwards. Se indica que los forwards poseen más masa adiposa y masa total que los backs. 
TABLA 1

Comparación de las características antropométricas de los grupos

\begin{tabular}{lccc}
\hline Variable & $\begin{array}{c}\text { Backs } \\
(\boldsymbol{n}=\mathbf{1 1})\end{array}$ & $\begin{array}{c}\text { Forwards } \\
(\boldsymbol{n}=\mathbf{1 1})\end{array}$ & Valor p \\
& $\mathbf{X} \pm \mathbf{D S}$ & $\mathbf{X} \pm \mathbf{D S}$ & \\
\hline Masa total (kg) & $80,02 \pm 2,97 *$ & $97,37 \pm 2,72 *$ & .012 \\
\hline Estatura (cm) & $180 \pm 0,01$ & $181 \pm 0,01$ & .882 \\
\hline Masa adiposa (\%) & $13,27 \pm 1,41^{*}$ & $19,21 \pm 1,37 *$ & .019 \\
\hline Masa libre de grasa (kg) & $68,54 \pm 2,62 *$ & $78,32 \pm 1,37 *$ & .005 \\
\hline
\end{tabular}

Nota $\mathrm{X}=$ promedio, $\mathrm{DS}=$ desviación estándar. ${ }^{*}$ La diferencia es significativa en el nivel 0,05

En la tabla 2, se encontraron diferencias estadísticamente significativas $\left({ }^{*}\right)$ en las variables $\mathrm{CMJ}(\mathrm{p}=.005)$ entre los grupos de backs y forwards. Esto indica que los backs posen más condición neuromuscular que los forwards, en función de una menor masa de grasa.

TABLA 2

Comparación de los test de potencia

\begin{tabular}{lccc}
\hline & $\begin{array}{c}\text { Backs } \\
(\boldsymbol{n}=\mathbf{1 1})\end{array}$ & $\begin{array}{c}\text { Forwards } \\
(\boldsymbol{n}=\mathbf{1 1})\end{array}$ & Nivel p \\
& $\mathbf{X} \pm \mathbf{D S}$ & $\mathbf{X} \pm \mathbf{D S}$ & \\
& $36,02 \pm 1,59^{*}$ & $28,73 \pm 1,74^{*}$ & .005 \\
\hline CMJ (cm) & $2,08 \pm 0,07$ & $1,90 \pm 0,09$ & .114 \\
\hline Salto horizontal $(\mathbf{c m})$ & $6,81 \pm 0,27$ & $6,21 \pm 0,32$ & .085 \\
\hline Salto horizontal triple $(\mathbf{c m})$ & & & \\
\hline
\end{tabular}

Nota $\mathrm{X}=$ promedio, $\mathrm{DS}$ = desviación estándar, $\mathrm{CMJ}=$ Counter Movement Jump

\section{Discusión}

El objetivo de este estudio fue comparar las variables antropométricas y de potencia de miembros inferiores (PMI) en una muestra de jugadores de rugby-7. Los resultados evidenciaron que los jugadores de la posición back tienen menor masa total, masa adiposa y masa libre de grasa, en relación con los forwards. Esto se debe a la especificidad de la función que exige de estos jugadores, con una estructura corporal más desarrollada (Dacres-Mannings, Rochester y Frail, 2001; Scott, Roe, Coats y Peipoli, 2003). Los estudios de Ezequiel et al. (2013), Lopes et al. (2011), Sant'Anna (2010) y Trindade (2010), en el Rugby Unión, evidenciaron diferencias significativas en relación con la masa, masa adiposa, masa libre de grasa de los backs en relación 
con las de los forwards. Estas distinciones son atribuidas por la posición que cada jugador ejerce dentro del campo. Es preciso tomar en cuenta que los forwards se encuentran, a lo largo de todo el partido, en mayor contacto con los oponentes, por lo que necesitan una mayor masa corporal y, consecuentemente, más masa muscular para más resistencia; mientras, los backs necesitan más agilidad y velocidad, lo que les hace un grupo con menor porcentaje de grasa y, en efecto, de mayor cantidad de masa libre de grasa (Sant'Anna, 2010).

Se encontraron diferencias significativas en la variable PMI, en la altura del CMJ. De Lacey et al. (2014), McMahon, Murphy, Rej y Comfort (2016) y Trindade (2010) también constataron que los backs presentaron mejor desempeño comparado con el de los forwards; al parecer, en la literatura la masa magra interfiere directa y positivamente en el desempeño en el CMJ en jugadores de rugby. En el estudio de Sant'Anna (2010) se utilizó la prueba de Wingate, lo cual representa una limitación, ya que se realiza en un cicloergómetro y esto no es lo real de la modalidad. Por eso, se debe trabajar con saltos, lo más cercano a la realidad del deporte. Cambios en los procedimientos pueden afectar directamente los resultados; tal es el caso de una disciplina como el rugby, en la que los forwards presentan mayor cantidad de masa adiposa, pues el exceso de grasa corporal provoca un aumento en la masa corporal. Esto último resulta en una pérdida de desempeño deportivo en fundamentos que involucran velocidad y potencia explosiva, como los saltos, ya que la aceleración es igual a la fuerza dividida por la masa (Duthie, Pyne y Hooper, 2003; Lorenzo y Chamorro, 2004).

La PMI es una de las capacidades que más se manifiestan en los jugadores de rugby, debido a las situaciones de juego que involucran patadas y carreras de alta intensidad (Bompa y Claro, 2015). Por esta especificidad de las funciones, los backs necesitan mayor PMI en relación con los forwards, pues se utiliza en constantes sprints para buscar ganar ventaja en los desmarques, dribles y disputas de velocidad (Cruz-Fereira y Ribeiro, 2013). Este estudio aporta conocimientos que pueden ser útiles para los entrenadores en este deporte, puesto que pueden utilizar esta información con el afán de adaptar el tipo de entrenamiento requerido para sus jugadores, mejorando el trabajo efectuado con ellos.

\section{ConCLuSión}

El presente trabajo demostró que los jugadores de la posición back tienen una mayor potencia de miembros inferiores, en relación con los de la posición forward, lo que se justifica por la disposición de los movimientos deportivos. Como limitación, el estudio apunta que la muestra compuesta por universitarios y el tiempo de entrenamiento de los practicantes eran menores en comparación con los de jugadores de nivel profesional. Cabe resaltar la importancia de evaluar a los practicantes con protocolos más específicos para el rugby-7.

\section{REFERENCIAS}

Bompa, T. y Claro, F. (2009). Periodization in Rugby. 1st ed. Londres: Meyer \& Meyer Sport.

Bosco, C. y Riu, J. M. P. (1994). La valoración de la fuerza con el test de Bosco. Barcelona, España: Paidotribo.

Caneviski, J. I. M., Crepaldi, J. R. y Fernandes, E. V. (2017). Influência do Aquecimento no Desempenho do Teste de Salto Horizontal em Jovens Adultos. Journal of Health Sciences, 19(3), 149-153. DOI: http:// dx.doi.org/10.17921/2447-8938.2017v19n3p149-153

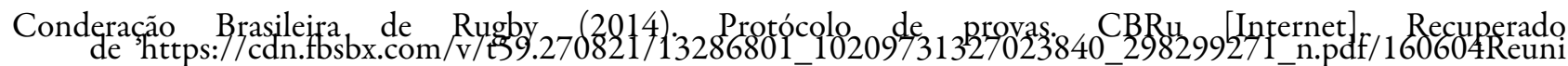
\%C3\%A3oEstrat\%C3\%A9gicaAberta1.pdf? $\mathrm{oh}=1 \mathrm{e} 12 \mathrm{efebe} 23 \mathrm{e} 21743 \mathrm{e} 036 \mathrm{c} 66 \mathrm{~d} 7 \mathrm{ab} 52 \mathrm{~d} 8 \&$ oe $=5759 \mathrm{D} 66 \mathrm{~B} \& \mathrm{dl}=1$

Cronin, J. y Sleivert, G. (2005). Challenges in understanding the influence of maximal power training on improving athletic performance. Sports Medicine, 35(3), 213-234. PMID: 15730337 
Cruz-Ferreira, A. M. D. y Ribeiro, C. A. F. (2013). Perfil antropométrico e fisiológico dos jogadores de rugby portugueses-Parte I: comparação entre atletas de diferentes grupos posicionais. Rev. Bras. Med. Esporte, 19(1), 48-51. DOI: http://dx.doi.org/10.1590/S1517-86922013000100010

Dacres-Mannings, S., Rochester, S. y Frail, H. (2001). Anthropometric profiles of Australian Rugby Institute. Club and State Level Rugby Union Players.

De Lacey, J., Brughelli, M. E., McGuigan, M. R. y Hansen, K. T. (2014). Strength, speed and power characteristics of elite rugby league players. J. Strength Cond. Res., 28(8), 2372-2375. DOI: 10.1519/ JSC.0000000000000397

Dinardi, R. R., Andrade, C. R. y Ibiapina, C. (2017). Effect of the Airmax Internal Nasal Dilator on Peak Nasal Inspiratory Flow, Aerobic Capacity, and Rating of Perceived Exertion in Healthy Rugby Players. Journal of Exercise Physiology Online, 20(1) 92-101. Recuperado de https://www.asep.org/asep/asep/ JEPonlineFEBRUARY2017_Dinardi.pdf

Dobbin, N., Richard, H., Jamie, H. y Craig, T. (2017). Validity of a Jump Mat for assessing Countermovement Jump Performance in Elite Rugby Players. Int. J. Sports Med., 38(2), 99-104. DOI: 10.1055/s-0042-118313

Duthie, G., Pyne, D. y Hooper, S. (2003). Applied physiology and game analysis of rugby union. Sports Medicine, 33(13), 973-991. PMID: 14606925

Ezequiel, B., Balbo, G. y Sousa, V. (2013). Perfil antropométrico dos praticantes de Rugby da região do abc. Tese. Faculdades Integradas de Santo André.

Haff, G. G. y Nimphius, S. (2012). Training principles for power. Strength \& Conditioning Journal, 34(6), 2-12. DOI: http://dx.doi.org/10.1519/SSC.0b013e31826db467

Haunsicker P. y Reiff, G. (1976). AAHPER. 3rd ed. Washington: AAHPER Publications. Recuperado de https:// files.eric.ed.gov/fulltext/ED120168.pdf

Jackson, A. S. y Pollock, M. L. (1978). Generalized equations for predicting body density of men. British Journal of Nutrition, 40(3), 497-504. DOI: https://doi.org/10.1079/BJN19780152

Leite, M., Zanetti, H., Lourenço, C., Mota, G., Sasaki, J. y Mendes, E. (2016). Diferenças das capacidades de desempenhos entre atletas amadores de rugby a partir da posição exercida em campo. Arquivos Ciências do Esporte, 4(1), 16-19. Recuperado de http://seer.uftm.edu.br/revistaeletronica/index.php/aces/article/ view/1120/1993

Lopes, A. L., Sant'Ana, R. T., Baroni, B. M., Santos Cunha, G., Radaelli, R., Oliveira, Á. R. y Souza Castro, F. (2011). Perfil antropométrico e fisiológico de atletas brasileiros de "rugby". Revista Brasileira de Educação Física e Esporte, 25(3), 387-395. Recuperado de http://www.scielo.br/pdf/rbefe/v25n3/v25n3a04.pdf

Lorenzo, M. G. y Chamorro, R. P. G. (2004). Índice de masa corporal y composición corporal: Un estudio antropométrico de 2500 deportistas de alto nivel. Lecturas: Educación Física y Deportes, (76), 34. Disponible en: http://www.efdeportes.com/efd76/antrop.htm

Loturco, I., Pereira, L. A., Moraes, J. E., Kitamura, K., Abad, C. C. C., Kobal, R. y Nakamura, F. Y. (2017). Jump-Squat and Half-Squat Exercises: Selective Influences on Speed-Power Performance of Elite Rugby Sevens Players. PLoS One, 12(1), 1-11. DOI: 10.1371/journal.pone.0170627

McMahon, J. J., Murphy, S., Rej, S. J. E. y Comfort, P. (2017). Countermovement-Jump-Phase Characteristics of Senior and Academy Rugby League Players. Int. J. Sports Physiol. Perform., 12(6), 803-811. DOI: 10.1123/ ijspp.2016-0467

Monteiro, E. R., Mendes, A., Paz, G. A., Miranda, H. L. y Lima, V. P. (2015). Perfil cardiorrespiratório entre atacantes e defensores de uma equipe profissional de rugby. Revista Brasileira de Ciência e Movimento, 24(1), 94-100. Recuperado de https://portalrevistas.ucb.br/index.php/RBCM/article/view/5871/4264

Navarro-Zurita, L., Gálvez, J., López, S. y Suárez-Arrones, L. (2017). Juegos Reducidos en Rugby: diferencias entre el uso o no de contactos y distintos espacios de interacción. RICYDE. Revista Internacional de Ciencias del Deporte, 13(49). DOI: https://doi.org/10.5232/ricyde

Roe, G. A., Darrall-Jones, J. D., Till, K. y Jones, B. (2016). Preseason changes in markers of lower body fatigue and performance in young professional rugby union players. European Journal of Sport Science, 16(8), 981-988. DOI: https://doi.org/10.1080/17461391.2016.1175510 
Sant'anna, R. (2010). Características fisiológicas e antropométricas de jogadores amadores de Rugby. Tese. Universidade Federal do Rio Grande do Sul.

Scott, A. C., Roe, N., Coats, A. J. y Piepoli, M. F. (2003). Aerobic exercise physiology in a professional rugby union team. International Journal of Cardiology, 87(2), 173-177. DOI: https://doi.org/10.1016/ S0167-5273(02)00211-5

Sousa, N. M. F., Stinguel, H., Souza Mairink, R., Baia, D. P., Bertucci, D. R. y Martins, R. A. S. (2016). Perfil antropométrico e aeróbio de jogadoras de uma equipe de rugby sevens: diferenças entre posiçôes táticas. RBPFEX-Revista Brasileira de Prescrição e Fisiologia do Exercício, 10(58), 282-289. Recuperado de http:// www.rbpfex.com.br/index.php/rbpfex/article/view/934/789

Trindade, C. (2010). Potência de jogadores de rugby determinado pelo R.A.S.T teste. Tese. Universidade Estadual de Maringá.

Worldrugby.org. (2014). Strategic Plan [online]. Recuperado de http://www.worldrugby.org/strategic-plan

\section{BY-NC-ND}

